\title{
The dark exciton in a quantum dot- a novel bright qubit with very long coherence time
}

\author{
E. Poem ${ }^{1}$, Y. Kodriano ${ }^{1}$, N. H. Lindner ${ }^{2}$, B. D. Gerardot ${ }^{3}$, P.M. Petroff ${ }^{4}$ and D. Gershoni ${ }^{1}$ \\ ${ }^{I}$ The Department of Physics and the Solid State Institute, Technion - Israel Institute of Technology, 32000 Haifa, Israel \\ ${ }^{2}$ Institute of Quantum Information, Caltech, Pasadena, CA, 91125, USA \\ ${ }^{3}$ Physics Department, Heriot-Watt University, Edinburgh EH14 4AS, UK \\ ${ }^{4}$ Material Department, University of California Santa Barbara, CA, 93106, USA \\ dg@physics.technion.ac.il
}

\begin{abstract}
We demonstrate for the first time that the quantum dot confined dark exciton is a natural, coherent and long-lived qubit. We optically "write" its spin state and successfully "read" its subsequent coherent evolution.

(C)2010 Optical Society of America

OCIS Codes: (270.5585) Quantum information and processing; (300.6470) Spectroscopy, semiconductors
\end{abstract}

The fundamental optical excitation of a semiconductor quantum dot (QD) is an electron-heavy-hole pair with antiparallel spins - the 'bright' exciton $\left(\mathrm{X}^{0}\right)$. This two-level system was proposed as a realization of a qubit and a logical gate [1] and its coherent properties and functionality were experimentally demonstrated [2-6]. Its advantage over the electron and/or hole spin, is in its neutrality, and thus insensitivity to its electrostatic environment. Its disadvantage is its short lifetime due to radiative decay.

A second type of exciton is that in which the electron and the heavy-hole spins are oriented parallel to each other. In this case, a single photon cannot take the total spin, and the pair is optically inactive, forming a 'dark' exciton. Like the bright one, the dark exciton is neutral. Its lifetime, however, is orders of magnitude longer.

Due to the exchange interaction between the electron and the hole, dark excitons are lower in energy than bright ones. Thus, at low temperatures, they are protected from a spin flip of one of their constituents. Their interaction with the nuclear spins, the main source of spin decoherence in semiconductors, is therefore comparable to that of heavy holes [7]. This constitutes substantial advantage for their implementation as qubits. Moreover, since the electron-hole exchange interaction acts transversally to the nuclear fields, the dark exciton's coherence properties are further improved in comparison with that of heavy holes. Finally, since the electron and hole g-factors are opposite in sign [8], the dark exciton interaction with the nuclear field is further reduced, compared with that of the electron, hole or the bright exciton. Therefore, the dark exciton's coherence properties are expected to be better than those of either, the electron, the hole or the bright exciton.

The main obstacle for utilizing the QD-confined dark exciton as a qubit is its optical inaccessibility. Yet, we succeeded to efficiently access this novel, coherent qubit, as we will demonstrate, in this post deadline submission.

A key to our success is the identification of a new metastable biexciton state which allows us to circumvent the difficulty in accessing the dark exciton. This metastable state can be excited quite efficiently by using two resonantly tuned, co-circularly polarized laser pulses. As explained in Fig. 1(a) below, we succeed in "writing" the dark exciton spin state $|+2\rangle(|-2\rangle)$ by the detection of a right (left) circularly polarized photon emitted from this metastable biexciton state $\left(\mathrm{XX}_{\mathrm{T} \pm 3}^{0}\right)$ in which the two heavy holes are in a triplet state with parallel spin projections, blockaded from relaxation to the singlet ground state.

Due to the short range exchange interaction between the electron and the hole, the \pm 2$\rangle$ spin states are not eigenstates, but rather coherent superpositions of the dark exciton's eigenstates - the symmetric and anti-symmetric spin configurations: $|S\rangle=1 / \sqrt{ } 2(|+2\rangle+|-2\rangle),|A\rangle=1 / \sqrt{ } 2(|+2\rangle-|-2\rangle)$. Consequently, the dark exciton's spin starts to precess [Fig. 1(b)] in a frequency given by the exchange interaction energy divided by the Planck constant.

The dark exciton does not recombine radiatively. Thus, optical readout of its spin state requires an additional mediating process. We use charge carriers' tunneling into the QD [Fig.1(c, d)], for that purpose. Charge tunneling can be induced externally, at will [9]. In our demonstration, however, we take advantage of spontaneous charge tunneling due to optical deionization of impurities near by the QD [10]. We control the charging rate by tuning the energy and the intensity of the exciting light [10].

Pauli's exclusion principle dictates that an electron (hole) may tunnel into the QD forming thus a negatively (positively) charged exciton, $\mathrm{X}^{-1}\left(\mathrm{X}^{+1}\right)$, if and only if its spin is anti parallel to that of the dark exciton's electron 


\section{QPDA9.pdf}

(hole). Therefore, the tunneling probability depends on the temporal evolution of the dark exciton's spin direction. After the carrier tunnels, the resulting charged exciton decays radiatively by emitting a photon whose circular polarization depends on the spin direction of the unpaired carrier at the tunneling time [Figs. 1(c, d)].

We demonstrate these ideas experimentally, using time resolved, polarization sensitive, intensity correlation measurements between the $\mathrm{XX}_{\mathrm{T} \pm 3}^{0}$ spectral line and either the $\mathrm{X}^{+1}$ or the $\mathrm{X}^{-1}$ spectral lines. The polarization in which the $\mathrm{XX}_{\mathrm{T} \pm 3}^{0}$ photon is detected determines the state in which the spin of the dark exciton is prepared, or "written". The polarization in which the $\mathrm{X}^{-1}$ (or the $\mathrm{X}^{+1}$ ) photon is detected determines the direction upon which the dark exciton spin is projected ("readout"), at the charge carrier's tunneling time.

The measured, background subtracted, degree of circular polarization of the charged exciton photon as a function of time after the detection of a circularly polarized photon from the $\mathrm{XX}_{\mathrm{T} \pm 3}^{0}$ metastable biexciton is given in Fig. 2 for both the $\mathrm{X}^{+1}$ (hole tunneling - red circles) and $\mathrm{X}^{-1}$ (electron tunneling - blue squares). The polarization degree measures the projection of the dark exciton's spin state on the growth axis, at the tunneling time.

From this measurement, we obtain the precession period $(2.9 \pm 0.2 \mathrm{nsec})$. This period yields $1.4 \pm 0.1 \mu \mathrm{eV}$ for the dark exciton's fine structure. Our work is therefore also the first to directly measure the short range electron-hole exchange interaction in this technologically important material system [8]. The similarity between the dark exciton fine structure and the radiative width of the metastable biexciton $(\sim 1.3 \mu \mathrm{eV}$ from its $\sim 1 \mathrm{nsec}$ decay time $)$ is in perfect agreement with the measured degree of polarization ( $50 \%$, see Fig. 2). Clearly, the state maintains its coherence within the entire temporal window set by our measurement method. We therefore demonstrate, for the first time, a novel, long-lived, with long coherence time solid state qubit. We also provide methods for easily accessing this coherent solid state qubit by external probes.

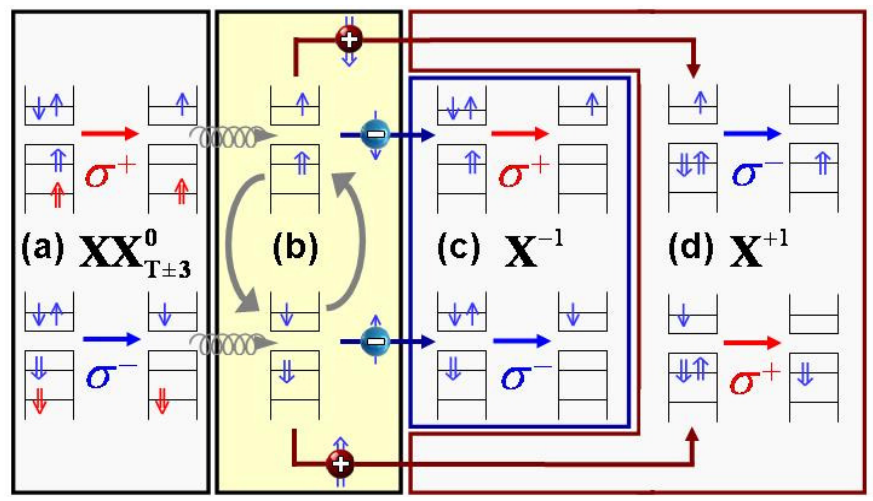

FIG. 1: (a) "Writing" the dark exciton in the spin state $|+2\rangle(|-2\rangle)$ by detecting a right- (left-) hand circularly polarized metastable biexciton $\mathrm{XX}_{\mathrm{T}+3(-3)}^{0}$ photon indicated by a red (blue) arrow. (b) Fast non-radiative decay of the hole to its ground state (curly arrows) and precession of the dark-exciton's spin induced by the short range e-h exchange interaction. (c) Readout of the dark exciton's state by electron tunneling and subsequent emission of a circularly polarized photon from the negatively charged exciton. (d) As in (c), but for hole tunnelling. Note the opposite polarization selection rules for the two cases.

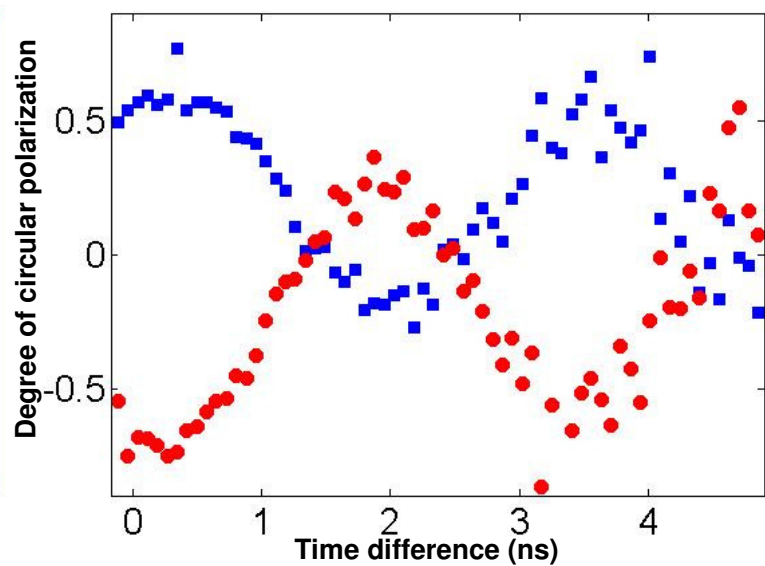

FIG. 2: Red circles (blue squares) represent the measured degree of circular polarization of the positively (negatively) charged exciton photon polarization as a function of time after the detection of a circularly polarized photon from the $\mathrm{XX}_{\mathrm{T} \pm 3}^{0}$ metastable biexciton spectral line.

[1] E. Biolatti et al., "Quantum information processing with semiconductor macroatoms", Phys. Rev. Lett. 85, 5647, (2000).

[2] N. H. Bonadeo et al., "Coherent optical control of the quantum state of a single quantum dot", Science 282, 1473, (1998).

[3] A. Zrenner et al., "Coherent properties of a two-level system based on a quantum-dot photodiode", Nature 418, 612, (2002).

[4] X. Li et al., "An all optical quantum gate in a semiconductor quantum dot", Science 301, 809, (2003).

[5] N. Akopian et al., "Entangled photon pairs from semiconductor quantum dots", Phys. Rev. Lett. 96, 130501, (2006).

[6] S. J. Boyle et al., "Two-qubit conditional quantum-logic operation in a single self-assembled quantum dot", Phys. Rev. B. 78, 075301, (2008).

[7] J. Fischer, et al., "Spin decoherence of a heavy hole coupled to nuclear spins in a quantum dot", Phys. Rev. B. 78, 155329, (2008).

[8] M. Bayer et al., "Fine structure of neutral and charged excitons in self-assembled In(Ga)As/(Al)GaAs quantum dots", Phys. Rev. B 65, 195315 (2002).

[9] R. J. Warburton, et al., "Optical emission from a charge tunable quantum ring", Nature 405, 926 (2000).

[10] A. Hartmann, et al., "Few-particle effects in semiconductor quantum dots: observation of multicharged excitons", Phys. Rev. Lett. 84, 5648, (2000). 